the $\mathrm{L}_{\mathrm{D}}$ subtype of voltage-gated $\mathrm{Ca}^{2+}$ channels in pancreatic $\beta$ cells. Proc. Natl. Acad. Sci. U. S. A. 96:10164-10169.

14. Ji, J., et al. 2002. Modulation of L-type $\mathrm{Ca}^{2+}$ channels by distinct domains within SNAP-25. Diabetes. 51:1425-1436.

15. Wiser, O., et al. 1999. The voltage sensitive $\mathrm{L}_{\mathrm{C}}$-type $\mathrm{Ca}^{2+}$ channel is functionally coupled to the exocytotic machinery. Proc. Natl. Acad. Sci. U. S. A. 96:248-253.

16. Straub, S.G., and Sharp, G.W. 2004. Hypothesis: one rate-limiting step controls the magnitude of both phases of glucose-stimulated insulin secretion. Am. J. Physiol. Cell Physiol. 287:C565-C571.

17. Berggren, P.-O., et al. 2004. Removal of $\mathrm{Ca}^{2+}$ channel $\beta 3$ subunit enhances $\mathrm{Ca}^{2+}$ oscillation frequency and insulin exocytosis. Cell. 119:273-284.

18. Li, W., Llopis, J., Whitney, M., Zlokarnik, G., and Tsien, R.Y. 1998. Cell-permeant caged InsP3 ester shows that $\mathrm{Ca}^{2+}$ spike frequency can optimize gene expression. Nature. 392:936-941.

19. Bonner-Weir, S. 2000. Perspective: Postnatal pancreatic beta cell growth. Endocrinology. 141:1926-1929.

20. Thore, S., Dyachok, O., and Tengholm, A. 2004 Oscillations of phospholipase $\mathrm{C}$ activity triggered by depolarization and $\mathrm{Ca}^{2+}$ influx in insulin-secret- ing cells. J. Biol. Chem. 279:19396-19400.

21. Fagan, K.A., Graf, R.A., Tolman, S., Schaack, J., and Cooper, D.M. 2000. Regulation of a $\mathrm{Ca}^{2+}$-sensitive adenylyl cyclase in an excitable cell. Role of voltagegated versus capacitative $\mathrm{Ca}^{2+}$ entry. J. Biol. Chem. 275:40187-40194.

22. Parker, P.J., and Murray-Rust, J. 2004. PKC at a glance. J. Cell. Sci. 117:131-132.

23. Arkhammar, P., et al. 1994. Protein kinase C modulates the insulin secretory process by maintaining a proper function of the $\beta$-cell voltage-activated $\mathrm{Ca}^{2+}$ channels. J. Biol. Chem. 269:2743-2749.

24. Eliasson, L., et al. 1996. PKC-dependent stimulation of exocytosis by sulfonylureas in pancreatic $\beta$ cells. Science. 271:813-815.

25. Zaitsev, S.V., Efendic, S., Arkhammar, P., Bertorello, A.M., and Berggren, P.-O. 1995. Dissociation between changes in cytoplasmic free $\mathrm{Ca}^{2+}$ concentration and insulin secretion as evidenced from measurements in mouse single pancreatic islets. Proc. Natl. Acad. Sci. U. S. A. 92:9712-9716.

26. Mendez, C.F., et al. 2003. Rapid association of protein kinase $C \varepsilon$ with insulin granules is essential for insulin exocytosis. J. Biol. Chem. 278:44753-44757.

27. Perander, M., Bjorkoy, G., and Johansen, T. 2001. Nuclear import and export signals enable rapid nucleocytoplasmic shuttling of the atypical protein kinase C $\lambda$. J. Biol. Chem. 276:13015-13024.

28. Harris, T.E., Persaud, S.J., and Jones, P.M. 1996. Atypical isoforms of $\mathrm{pKc}$ and insulin secretion from pancreatic beta-cells: evidence using Go 6976 and Ro 31-8220 as Pkc inhibitors. Biochem. Biophys. Res. Commun. 227:672-676.

29. Furukawa, N., et al. 1999. Possible involvement of atypical protein kinase $\mathrm{C}(\mathrm{PKC})$ in glucose-sensitive expression of the human insulin gene: DNA-binding activity and transcriptional activity of pancreatic and duodenal homeobox gene-1 (PDX-1) are enhanced via calphostin C-sensitive but phorbol 12-myristate 13-acetate (PMA) and Go 6976-insensitive pathway. Endocr. J. 46:43-58.

30. Way, K.J., Chou, E., and King, G.L. 2000. Identification of PKC-isoform-specific biological actions using pharmacological approaches. Trends Pharmacol. Sci. 21:181-187.

31 . Hashimoto, N., et al. 2005. PKC $\lambda$ regulates glucoseinduced insulin secretion through modulation of gene expression in pancreatic $\beta$ cells. J. Clin. Invest. 115:138-145. doi:10.1172/JCI200522232.

32. Rorsman, P., Berggren, P.-O., Bokvist, K., and Efendic, S. 1990. ATP-regulated $\mathrm{K}^{+}$channels and diabetes mellitus. News Physiol. Sci. 5:143-147.

\title{
A new mechanism of BRAF activation in human thyroid papillary carcinomas
}

\author{
Alfredo Fusco, ${ }^{1,2}$ Giuseppe Viglietto, ${ }^{1}$ and Massimo Santoro ${ }^{1}$ \\ 1Dipartimento di Biologia e Patologia Cellulare e Molecolare, Istituto di Endocrinologia ed Oncologia Sperimentale del CNR, \\ Facoltà di Medicina e Chirurgia di Napoli, Università degli Studi di Napoli "Federico II," Naples, Italy. \\ ${ }^{2}$ NOGEC (Naples Oncogenomic Center)-CEINGE, Biotecnologia Avanzate, Naples, Italy.
}

\begin{abstract}
In this issue of the JCI, Ciampi et al. report the identification of a novel oncogene in patients affected by radiation-associated thyroid papillary carcinomas (see the related article beginning on page 94). This oncogene derives from a paracentric inversion of the long arm of chromosome 7 , which results in an in-frame fusion of the $\mathrm{N}$-terminus of the A-kinase anchor protein 9 (AKAP9) gene with the $\mathrm{C}$-terminal catalytic domain (exons 9-18) of the serine-threonine kinase BRAF. The resulting AKAP9-BRAF fusion protein shows constitutive kinase activity, and it is able to transmit mitogenic signals to the MAPK pathways and to promote malignant transformation of NIH3T3 cells.
\end{abstract}

The Chernobyl nuclear power plant accident in 1986 caused severe radioiodide contamination of several areas in Belarus, Ukraine, and western Russia, leading to high radioactive exposure of the thyroid gland among the general population, including children. Beginning in 1992, a sharp increase in the incidence of childhood thyroid tumors, predominantly papillary thyroid carcinomas (PTCs), was

Nonstandard abbreviations used: $A K A P 9, A$-kinase anchor protein 9; FA, follicular adenoma; FTC, follicular thyroid carcinoma; PTC, papillary thyroid carcinoma.

Conflict of interest: The authors have declared that no conflict of interest exists.

Citation for this article: $J$. Clin. Invest. 115:20-23 (2005). doi:10.1172/JCI200523987. reported. The molecular analysis of these tumors has provided a unique opportunity to study the mechanisms of radiationdependent carcinogenesis in humans (1).

In this issue of the JCI, Ciampi et al. (2) report the identification of a novel oncogene in PTCs that developed in irradiated patients after a short latency period. This oncogene derives from the in-frame fusion of the first 8 exons of the A-kinase anchor protein 9 (AKAP9) gene with the C-terminal-encoding region (exons 9-18) of the proto-oncogene BRAF. AKAP9-BRAF fusion results from a paracentric inversion [inv (7)(q21-22q34)] of the long arm of chromosome 7 (Figure 1). BRAF is a serinethreonine kinase involved in the transmis- sion of signals from membrane receptors and RAS small GTPases to MAPK (Figure 2). This pathway transduces mitogenic signals in response to the activation of tyrosine kinase receptors. The AKAP9-BRAF fusion event results in the loss of 2 BRAF regulatory domains, CR1 and CR2 (Figure 2), which exert autoinhibitory effects on the kinase activity of BRAF; CR1 includes the RAS-GTP binding domain (3). Accordingly, the AKAP9-BRAF recombination and the loss of CR1 results in a RAS-independent gain-of-function of BRAF that is able to induce transformation of NIH3T3 cells that become tumorigenic after injection into athymic mice.

\section{Oncogene activation in human PTCs}

The AKAP9-BRAF fusion event reported by Ciampi and coworkers (2) represents an additional example of oncogene formation due to chromosomal rearrangements in human PTCs. Other examples include RET/PTC and TRK-T oncogenes. RET/PTC, present in about $40 \%$ of human PTCs, are chimeric genes generated by the fusion of the $\mathrm{C}$-terminal catalytic domain of the RET 

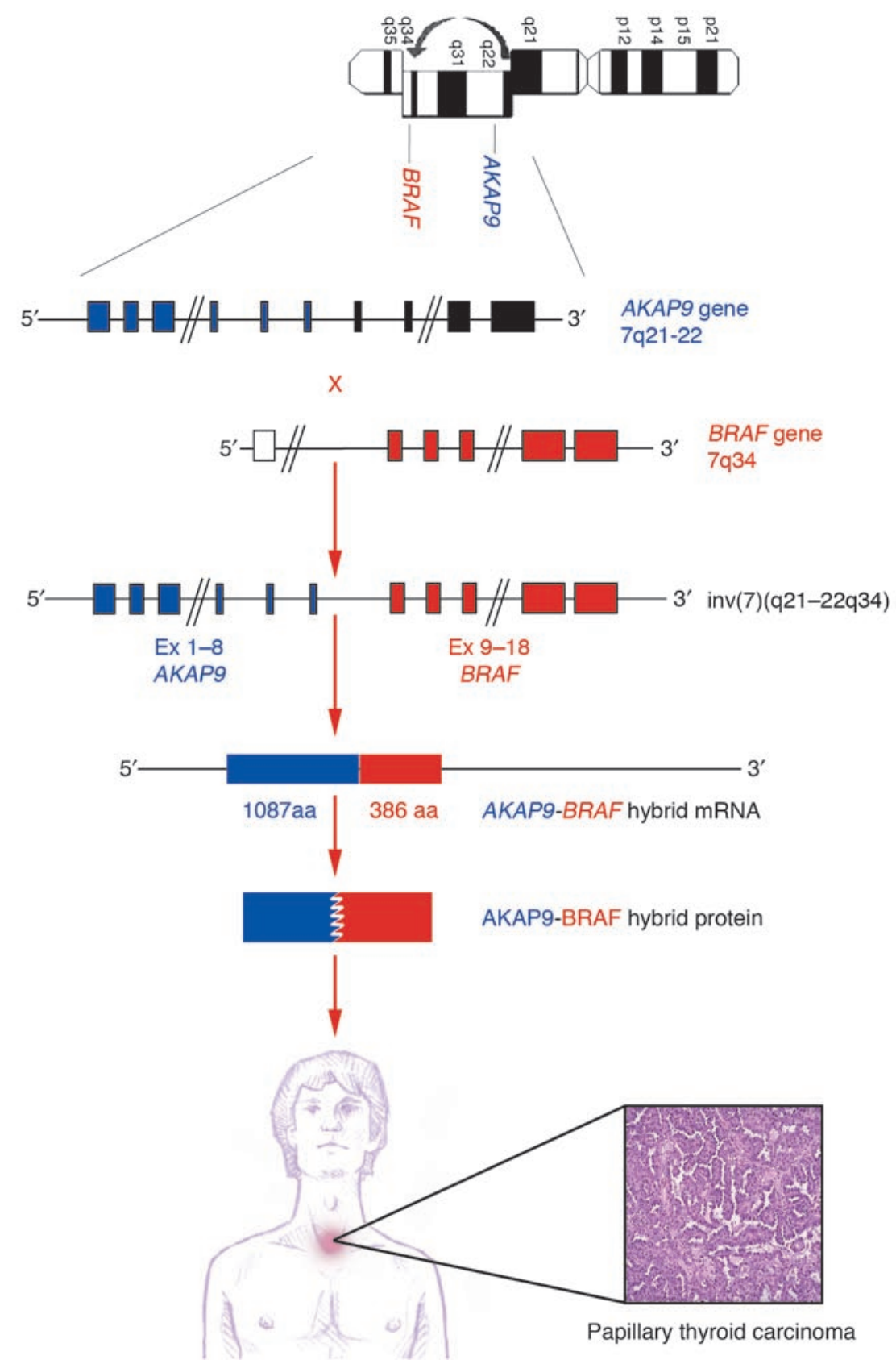

receptor tyrosine kinase with the N-terminal region encoded by heterologous genes. In the most prevalent variants, RET/PTC1 (4) and RET/PTC3 (5), the fusion occurs between RET and the H4 (also named D10S170) or RFG (also named Ele1/ARA70/ Ncoa4) genes. RET, H4, and RFG map to the long arm of chromosome 10 , and paracentric chromosomal inversions account for RET/PTC1 and RET/PTC3 generation (6). Similarly, TRK-T oncogenes, present in about $10 \%$ of human PTCs, are generated by structural rearrangements of the NTRK1 (also named TRKA) gene, coding for the high-affinity nerve growth factor (NGF) receptor. The TRK-T1 and TRK-T2 oncogenes are both generated by NTRK1 fusion with the TPR gene. NTRK1 and TPR genes are both located on chromosome 1 ; therefore, as is this case for AKAP9-BRAF fusion, the structural rearrangement is mediated by an intrachromosomal event (7).

\section{Chromosomal rearrangements are typical of PTCs}

In PTCs, the prevalence of gene rearrangements, particularly chromosomal inversions, is quite high. This is at odds with
Figure 1

Molecular mechanism of the chromosomal rearrangement generating the transforming AKAP9-BRAF oncogene in PTCs.

the low frequency of gene rearrangements in most epithelial tumors, where point mutations are prevalent (8). Interestingly, this tendency is not restricted to PTCs, since thyroid tumors of the follicular histotype, follicular thyroid carcinomas (FTCs), and follicular adenomas (FAs), are also characterized by chromosomal translocations. A [t(2;3)(q13;p25)] translocation involving the PAX 8 and PPAR 1 genes is a characteristic of a significant number of FTCs (9), whereas 2 chromosomal regions, $19 \mathrm{q} 13$ and $2 \mathrm{p} 21$, are frequently rearranged in FAs.

The study by Ciampi and coworkers (2) provides further evidence supporting the concept that chromosomal inversions represent the most typical molecular lesion in tumors occurring in Belarus and the surrounding region after the Chernobyl accident. In contrast, very rare point mutations have been detected in these tumors (10). The peculiar susceptibility of thyroid follicular cells to chromosomal rearrangements is remarkable. Previously, Yuri Nikiforov and coworkers (11) proposed an intriguing mechanism to explain how ionizing radiation can cause RET/PTC rearrangements and why this occurs preferentially in thyrocytes. They showed that while RET and $\mathrm{H} 4$ loci are about $30 \mathrm{Mb}$ apart in the linear map of chromosome 10 , they frequently juxtapose within the nuclei of thyroid cells but not in other cell types; this contiguity provides the structural basis for radiation-induced illegitimate nonhomologous recombination of the 2 genes (11). Another explanation was proposed to explain the peculiar susceptibility of thyroid follicular cells to chromosomal rearrangements. In vitro exposure of thyroid cells to ionizing radiation did not induce apoptosis but significantly increased DNA end-joining enzymatic activity (12). Thus, thyrocytes may respond to DNA damage with chromosomal alterations rather than cell death (6).

\section{BRAF activation is a common feature of both sporadic and post-Chernobyl PTCs}

$B R A F$ mutations have previously been implicated in the carcinogenesis of PTCs. Indeed, several groups have reported that 


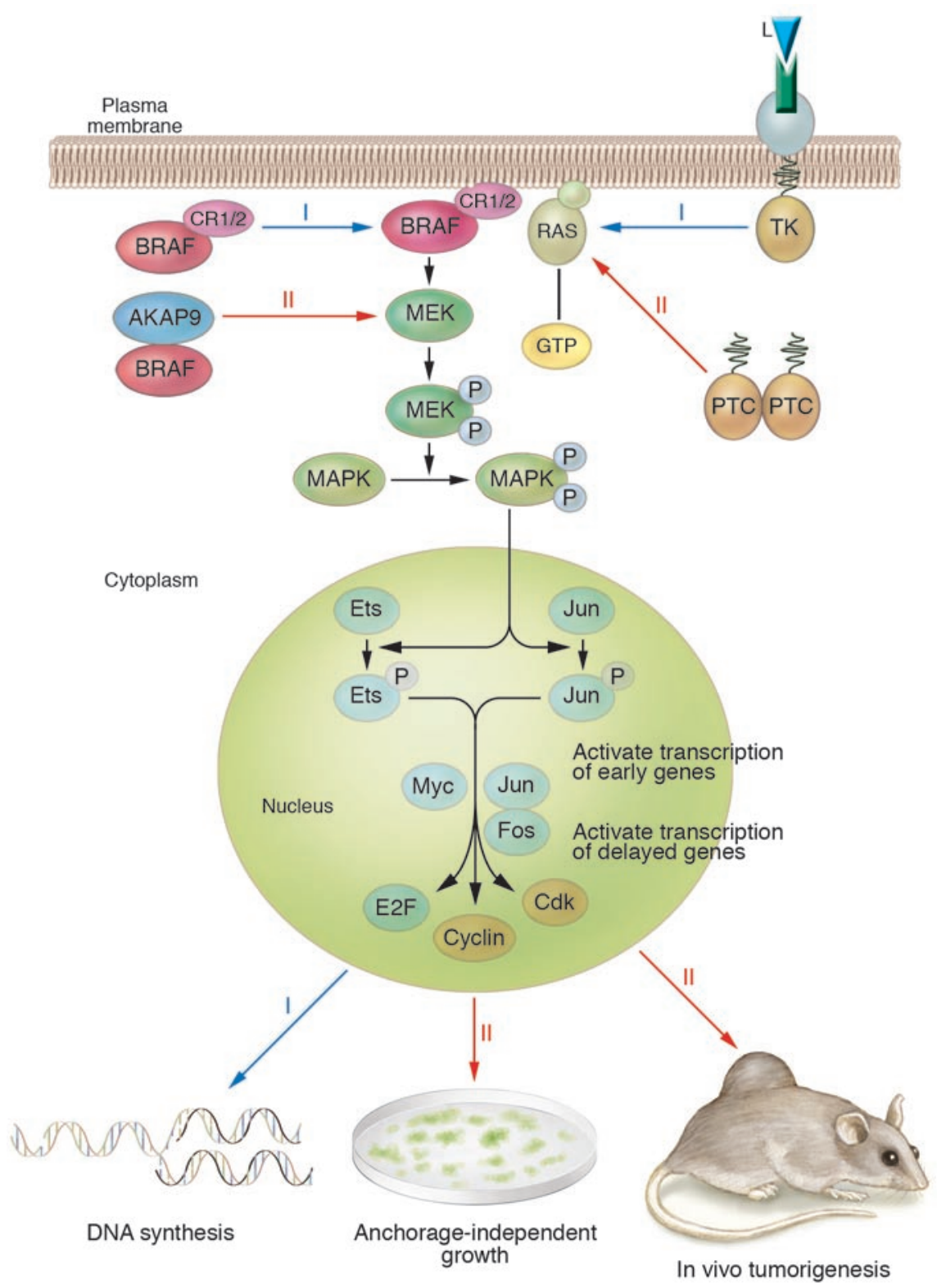

about $50 \%$ of adult PTCs harbor a specific point mutation (V600E, formerly designated V599E) in BRAF. In human cancer, $B R A F$ mutations only appear more frequently in melanomas (13). V600 maps to the activation segment of the BRAF kinase. Structural analysis has revealed that the V600E mutation constitutively activates BRAF by destabilizing the inactive form of the kinase, thereby shifting the equilibrium toward its active conformation (3). However, prior to this report by Ciampi et al. (2), BRAF activation was considered to be a feature of sporadic, non-radiation-associated PTCs; a negligible frequency of BRAF mutations having been detected in radiation-induced childhood PTCs (14-16).
In this context, this JCI study represents a major breakthrough in our knowledge of molecular events involved in PTC initiation. Indeed, it demonstrates that BRAF activation is a common feature of both sporadic and post-Chernobyl thyroid carcinomas and that it is the molecular mechanism underlying BRAF activation that differentiates the 2 types of tumors, with the higher prevalence of point mutations occurring in sporadic PTCs and intrachromosomal inversion responsible for a larger percentage of radiation-induced PTCs.

The identification of the AKAP9-BRAF oncogene in post-Chernobyl PTCs represents an important step forward in thyroid cancer research. Certainly, it will foster

\section{Figure 2}

The MAPK pathway. Once activated, tyrosine kinase (TK) receptors activate the monomeric G protein RAS (pathway I), which in turn binds the serine-threonine kinase BRAF by inducing a conformational change that allows its activation (pathway I) and hence activation of the MAPK pathway. The activation of the MAPK pathway results in DNA synthesis in response to the external mitogenic stimulus (pathway I). When the RET/PTC (pathway II) or the BRAF (pathway II) oncogenes are generated through chromosomal rearrangements, activation of the MAPK pathway becomes constitutive, and cells become able to proliferate indefinitely, to grow in an anchorage-independent manner, and to induce tumors after injection into athymic mice (pathway II). MEK, MAPK/ERK kinase; L, ligand.

new lines of research aimed at addressing still unanswered questions. Is the level of transforming ability exerted by the AKAP9$B R A F$ oncogene different from that exerted by $B R A F$ point mutants? Indeed, biological differences in the potency of these 2 oncogenic versions of the BRAF protein are expected, since childhood and adult tumors differ in some of their clinical characteristics (i.e., childhood PTCs are often characterized by distant metastases, which are rather rare in the adult).

Among the most intriguing issues to be addressed is the role played by the likely inactivation of the AKAP9 gene following the paracentric inversion [inv (7)(q21-22q34)] that generates the AKAP9-BRAF fusion protein. AKAP9 is capable of binding the type II regulatory subunit of the cAMPdependent PKA; intriguingly, the type I regulatory subunit - RI $\alpha$ - of PKA is fused to RET in the RET/PTC2 oncogene (17). Thus, the possibility that functional alterations in the PKA pathway are involved in the pathogenesis of PTCs carrying the AKAP9-BRAF or RET/PTC2 oncogenes deserves further investigation. It is also worth mentioning that loss-of-function mutations in the RI $\alpha$ regulatory subunit are responsible for the development of the Carney complex, a multiple endocrine neoplasia syndrome (18).

The report by Ciampi et al. (2) presents what we believe to be the first example of an intracellular effector in the MAPK pathway activated by recombination occurring in vivo and supports the concept that one specific signaling pathway that leads to MAPK activation plays a major role in the generation of PTCs. At this stage, this pathway is the most attractive target for novel pharmacological intervention in human PTCs. 
Address correspondence to: Alfredo Fusco, Dipartimento di Biologia e Patologia Cellulare e Molecolare c/o Istituto di Endocrinologia ed Oncologia Sperimentale del CNR, Facoltà di Medicina e Chirurgia di Napoli, Università degli Studi di Napoli "Federico II," via Pansini, 5, 80131 Naples, Italy. Phone: 0039-0813722857; Fax: 00390817463749; E-mail: afusco@napoli.com.

1. Williams, D. 2002. Cancer after nuclear fallout: lessons from the Chernobyl accident. Nat. Rev. Cancer. 2:543-549.

2. Ciampi, R., et al. 2005. Oncogenic AKAP9-BRAF fusion is a novel mechanism of MAPK pathway activation in thyroid cancer. J. Clin. Invest. 115:94-101. doi:10.1172/JCI200523237.

3. Dibb, N.J., Dilworth, S.M., and Mol, C.D. 2004 Switching on kinases: oncogenic activation of BRAF and the PDGFR family. Nat. Rev. Cancer. 4:718-727.

4. Grieco, M., et al. 1990. PTC is a novel rearranged form of the ret proto-oncogene and is frequently detected in vivo in human thyroid papillary carcinomas. Cell. 60:557-563.

5. Santoro, M., et al. 1994. Molecular characterization of RET/PTC3; a novel rearranged version of the RETproto-oncogene in a human thyroid papillary carcinoma. Oncogene. 9:509-516.

6. Pierotti, M.A. 2001. Chromosomal rearrangements in thyroid carcinomas: a recombination or death dilemma. Cancer Lett. 166:1-7.

7. Alberti, L., Carniti, C., Miranda, C., Roccato, E., and Pierotti, M.A. 2003. RET and NTRK1 proto-oncogenes in human diseases. J. Cell. Physiol. 195:168-186.

8. Futreal, P.A., et al. 2004. A census of human cancer genes. Nat. Rev. Cancer. 4:177-183.

9. Kroll, T.G., et al. 2000. PAX8-PPARgamma1 fusion oncogene in human thyroid carcinoma. Science. 289:1357-1360.

10. Fagin, J.A. 2004. Challenging dogma in thyroid cancer molecular genetics - role of RET/PTC and BRAF in tumor initiation. J. Clin. Endocrinol. Metab. 89:4264-4266

11. Nikiforova, M.N., et al. 2000. Proximity of chromosomal loci that participate in radiation-induced rearrangements in human cells. Science. 290:138-141.

12. Yang, T., et al. 1997. p53 induced by ionizing radiation mediates DNA end-jointing activity, but not apoptosis of thyroid cells. Oncogene. 14:1511-1519.

13. Kimura, E.T., et al. 2003. High prevalence of BRAF mutations in thyroid cancer: genetic evidence for constitutive activation of the RET/PTC-RAS-BRAF signaling pathway in papillary thyroid carcinoma. Cancer Res. 63:1454-1457.

14. Lima, J., et al. 2004. BRAF mutations are not a major event in post-Chernobyl childhood thyroid carcinomas. J. Clin. Endocrinol. Metab. 89:4267-4271.

15. Kumagai, A., et al. 2004. Low frequency of BRAFT1796A mutations in childhood thyroid carcinomas. J. Clin. Endocrinol. Metab. 89:4280-4284.

16. Nikiforova, M.N., et al. 2004. Low prevalence of BRAF mutations in radiation-induced thyroid tumors in contrast to sporadic papillary carcinomas. Cancer Lett. 209:1-6.

17. Bongarzone, I., et al. 1993. Molecular characterization of a thyroid tumor-specific transforming sequence formed by the fusion of ret tyrosine kinase and the regulatory subunit RI alpha of cyclic AMP-dependent protein kinase A. Mol. Cell. Biol. 13:358-366.

18. Kirschner, L.S., et al. 2000. Mutations of the gene encoding the protein kinase A type I-alpha regulatory subunit in patients with the Carney complex. Nat. Genet. 26:89-92.

\section{The promise of stem cells in Parkinson disease}

\section{J. William Langston}

Parkinson's Institute, Sunnyvale, California, USA.

\begin{abstract}
Neurotransplantation as a treatment for Parkinson disease reached the stage of human trials over 15 years ago, but the field, which is still in its infancy, has encountered a number of roadblocks since then, both political and scientific. With hope that stem cells may be used as a new source of dopaminergic neurons to replace the degenerating nerve cells in Parkinson disease looming, it is critical that we learn from the past as we work toward achieving new milestones aimed at making this new therapeutic strategy a reality. One of those milestones, which is an important translational step in the development of stem cell technology and the subject of a report in this issue of the $J C I$, involves transplanting new dopaminergic cell lines to a primate model of Parkinson disease (see the related article beginning on page 102).
\end{abstract}

The possibility of repairing the damaged human brain has been a dream of physicians and scientists for decades. Over time it has become obvious that Parkinson disease is a natural first when it comes to tackling this ambitious feat, primarily because the majority of the signs and symptoms appear to result from the progressive loss of cells in a small area known as the substantia nigra, which sits atop the brain stem. These cells make dopamine, which is delivered to a part of the basal ganglia known as the striatum; when nigral neurons die and striatal

Nonstandard abbreviations used: DA, dopaminergic.

Conflict of interest: The author has declared that no conflict of interest exists.

Citation for this article: J. Clin. Invest. 115:23-25 (2005). doi:10.1172/JCI200524012. dopamine diminishes, the signs and symptoms of Parkinson disease become manifest. Thus, replenishing missing neurons in a limited area of the brain should in theory reverse parkinsonism, making this an attractive approach. But the challenge of actually replacing injured and/or lost neurons in the adult human nervous system has proven to be a daunting task with far more bumps in the road, both political and scientific, than anyone would have anticipated.

\section{Neurotransplantation: trials and tribulations}

While stem cell therapy is very much in the forefront when approaches to brain repair and cell replacement therapy are being considered, there is already a substantial body of work in the Parkinson disease field involving neurotransplantation, including the use of both adult adrenomedullary tissue and human fetal mesencephalic tissue (which is rich in dopaminergic [DA] neurons); the lessons learned should not be ignored. While adrenomedullary transplantation proved to be something of a medical fiasco, with little efficacy and unacceptable morbidity and mortality, a number of the early open label trials with human fetal mesencephalic tissue appeared to be very promising. However, due to a ban on the use of federal funds for research utilizing human fetal tissue imposed by then-President Ronald Reagan, little work was done in this area until President Bill Clinton lifted this moratorium on his second day in office. Not long thereafter, 2 large controlled clinical trials aimed at using fetal human mesencephalic tissue transplanted to the striatum to treat Parkinson disease were launched with federal funding. However, to the surprise of many, both trials failed to show a significant clinical benefit based on their primary endpoint variables $(1,2)$ in spite of substantial evidence of graft survival based on both autopsy (3) and imaging studies. Unfortunately, a substantial subset of patients also developed persistent excessive movements known as dyskinesias (2). Dyskinesias are typically a consequence of long-term L-dopa therapy, but in the patients 